\title{
Seletividade de diferentes agrotóxicos usados na cultura da soja ao parasitoide de ovos Telenomus remus
}

\author{
Selectivity of different pesticides used in soybean to the eggs parasitoid Telenomus remus
}

\author{
Eduardo Lima do Carmo ${ }^{\mathrm{I}}$ Adeney de Freitas Bueno ${ }^{\mathrm{II}}$ Regiane Cristina Oliveira de Freitas Bueno ${ }^{\mathrm{I}}$ \\ Simone Silva Vieira ${ }^{\mathrm{III}}$ Alysson Luis Gobbi ${ }^{\mathrm{IV}}$ Fabrício Rodrigues Vasco $^{\mathrm{IV}}$
}

\section{RESUMO}

A preservação dos agentes de controle biológico no agroecossistema é indispensável para o sucesso do manejo integrado de pragas. Entretanto, o controle químico de pragas ainda é indispensável em diversas culturas. Nesse contexto, a seletividade dos agrotóxicos aos inimigos naturais deve ser sempre considerada na escolha do melhor produto. Portanto, este estudo verificou o impacto causado por diferentes agrotóxicos na emergência do parasitoide de ovos Telenomus remus Nixon (Hymenoptera: Scelionidae), quando aplicados nas fases de larva e pupa (posturas de 100 a 150 ovos de Spodoptera frugiperda (J.E. Smith) (Lepidoptera: Noctuidae) seis e 10 dias após o parasitismo, respectivamente). Três ensaios, com 11 tratamentos e cinco repetições, foram conduzidos em laboratório com inseticidas, fungicidas e herbicidas, respectivamente. Cada ensaio foi conduzido com 10 tratamentos de agrotóxicos e água como testemunha seletiva. Todos os produtos fitossanitários foram testados nas doses utilizadas na cultura da soja. Ovos de $\mathbf{S}$. frugiperda parasitados por T. remus foram imersos nos tratamentos por cinco segundos e, após secagem total, foram acondicionados em sacos plásticos até a emergência dos adultos dos parasitoides. A viabilidade do parasitismo foi avaliada, e a redução na emergência dos parasitoides foi classificada segundo as normas da International Organization for Biological Control (IOBC). Os resultados mostraram que os inseticidas do grupo dos reguladores de crescimento, como o flufenoxurom, diflubenzurom $e$ metoxifenozido e também os inseticidas imidacloprido + betaciflutrina e acefato, foram seletivos às fases imaturas do parasitoide. Espinosade foi classificado como inócuo para larvas de T. remus. Gama-cialotrina foi levemente nocivo para larvas e inócuo para pupas. Bifentrina e clorpirifós foram, respectivamente, levemente nocivo e nocivo para ambas as fases de desenvolvimento de $\mathbf{T}$. remus. Com relação aos herbicidas testados, 2,4-D, S-metolacloro, flumioxazina, dicloreto de paraquate + diurom, dicloreto de paraquate $e$ glifosato (Roundup Transorb ${ }^{\circledR}$ ) foram seletivos. Entretanto, glifosato + imazetapir, clomazona, glifosato (Gliz ${ }^{\circledR}$ ), glifosato (Roundup Ready ${ }^{\circledR}$ ) foram seletivos para a fase de larva $e$ levemente nocivos (classe 2) para a fase de pupa de T. remus. Resultados semelhantes foram obtidos com os fungicidas flutriafol + tiofanato metílico, carbenzadim, trifloxistrobina + tebuconazole e azoxistrobina + ciproconazole, que foram classificados como inócuos. Tiofanato-metílico, tebuconazole e epoxiconazole + piraclostrobina foram levemente nocivos para larvas e inócuos para pupas. Inversamente, epoxiconazole e azoxistrobina foram seletivos para a fase de larva e levemente nocivos para a fase de pupa do parasitoide. Flutriafol foi classificado como levemente nocivo para ambas as fases de $\mathbf{T}$. remus.

Palavras-chave: controle químico, controle biológico, IOBC, Glycine max, Scelionidae.

\section{ABSTRACT}

Biological control agent preservation is crucial to the integrated pest management success. However, chemical control is yet useful for crop production. Thus, pesticide selectivity to beneficial insects is a feature that must always be taken into account in order to choose the best chemical. Therefore, this research studied the impact caused by different pesticides in the egg parasitoid, Telenomus remus Nixon (Hymenoptera: Scelionidae) emergence, when sprayed on the larvae and pupae stage (Egg masses from 100 to 150 eggs of Spodoptera frugiperda (J.E. Smith) (Lepidoptera: Noctuidae) six and ten days after the parasitism, respectively). Three trials, with 11 treatments and 5 repetitions, were carried out in the laboratory with insecticides, fungicides and herbicides. Each test was carried out with 10 treatments of chemicals and water as control. All chemicals were tested at rates used in soybean crops. Eggs of $\mathbf{S}$. frugiperda parasitized by $\mathbf{T}$. remus, were immersed in the treatments for five seconds. After completely dry, they were

'Universidade de Rio Verde (FESURV), Rio Verde, GO, Brasil.

IEmbrapa-Soja, 86001-970, Londrina, PR, Brasil. E-mail: adeney@cnpso.embrapa.br. Autor para correspondência.

II'Universidade do Estado de Santa Catarina-UDESC/CAV, Lages, SC, Brasil.

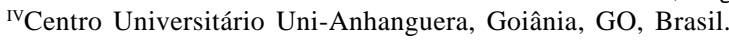


placed in plastic bags until the emergence of adults of parasitoids. The viability of parasitism was evaluated and the reduction in the emergence of the parasitoids classified according to the rules of the International Organization for Biological Control (IOBC). The results showed that the insect growth-regulating (IGR) insecticides flufenoxuron, diflubenzuron, methoxyfenozide as well as the insecticides imidacloprid + beta-cyfluthrin and acephate were harmless to the parasitoid immature stages. Spinosad was classified as harmless for larvae of T. remus. Gama-cyhalothrin was slightly harmful to larvae and harmless to pupa of T. remus. Bifenthrin and chlorpyrifos were, respectively, slightly harmful and harmful to both stages of development of $\boldsymbol{T}$. remus development. Regarding to the tested herbicides, 2,4-D, s-metolachlor, flumioxazin, paraquat dichloride + diuron, paraquat dichloride and glyphosate (Roundup Transorb ${ }^{\circledR}$ ) were harmless. However, glyphosate + imazethapyr, clomazone, glyphosate (Gliz $\left.{ }^{\circledR}\right)$, glyphosate (Roundup Ready ${ }^{\circledR}$ ) were harmless to the larvae stage and slightly harmful (class 2) to T. remus pupae. Similar results were obtained with the fungicides flutriafol + tiofanate, carbenzadin, tebuconazol + trifloxystrobin and azoxystrobin + ciproconazol which were classified as harmless. Tiofanate-metil, tebuconazol and epoxyconazol + pyraclostrobin were slightly harmful to larvae and pupae. Inversely, epoxyconazol and azoxystrobin were harmless to larvae and slightly harmful to pupae. Flutriafol was classified as slightly harmful to both development stages of $\mathbf{T}$. remus.

Key words: biological control, chemical control, IOBC, Glycine max, Scelionidae.

\section{INTRODUÇÃO}

O Brasil é um dos mais importantes produtores e exportadores de grãos no mundo. Tomando-se como exemplo a soja, Glycine max (Merril), de acordo com o levantamento da CONAB (2008), cerca de 21,16 milhões de hectares foram plantados na safra 2007/08. A cada ano, novas fronteiras são estabelecidas, em que, devido ao uso abusivo e errôneo de agrotóxicos, as relações de equilíbrio desses agroecossistemas são desestabilizadas, ocorrendo seleção de plantas daninhas, doenças e pragas resistentes e em altas populações, além da contaminação do homem e ambiente. Além disso, a explosão populacional de pragas anteriormente consideradas de importância secundária também pode ser considerada como outra consequência indesejável desse mau uso dos inseticidas (PALUMBO et al., 2001; DIEZ-RODRÍGUEZ \& OMOTO, 2001).

Para tentar minimizar esse quadro, tem-se enfatizado o emprego do controle biológico em programas de manejo integrado de insetos praga (MIP) (VAN LENTEREN \& BUENO, 2003), que preconiza a utilização de diversas táticas de controle de forma harmônica, visando a complementar o controle biológico natural a fim de manter as populações de pragas agrícolas abaixo do nível de dano econômico, conservando o ambiente e os artrópodes benéficos. Nesse contexto, o controle biológico é de grande importância para a sustentabilidade do sistema agrícola. Entretanto, o controle químico é considerado indispensável na cultura da soja. Os produtos mais adequados para serem utilizados no MIP são aqueles que combinam controle eficiente da praga com o menor impacto sobre a atividade dos inimigos naturais, sendo essa integração de produtos químicos com o controle biológico, na maioria dos casos, crucial para o sucesso do agronegócio (BUENO \& FREITAS, 2004). Inseticidas e acaricidas seletivos, bem como herbicidas, fungicidas e outros produtos químicos e biológicos, devem ser preferidos para que os inimigos naturais sejam preservados (BUENO et al., 2008a).

Dentre os inimigos naturais, destaca-se o parasitoide de ovos Telenomus remus, que é a espécie mais comum do gênero e já foi observada parasitando cinco diferentes espécies de Spodoptera spp. Cada fêmea, durante sua vida reprodutiva, produz cerca de 270 parasitoides (MORALES et al., 2000) e realiza a oviposição de apenas um ovo por hospedeiro, sendo o superparasitismo raro nessa espécie (CAVE, 2000). Esse parasitoide tem sido utilizado em larga escala em programas de MIP na Venezuela, por meio de liberações inundativas em áreas de milho, sendo obtidos índices de parasitismo de até 90\% (FERRER, 2001). Isso demonstra o grande potencial que essa espécie tem no controle biológico aplicado de lagartas Spodoptera spp. Lagartas desse gênero, principalmente as espécies $\boldsymbol{S}$. eridania e S. cosmioides, têm ocorrido na soja, nas últimas safras, ocasionando prejuízos econômicos, principalmente na região Centro-Oeste, o que pode estar relacionado com a eliminação de seus inimigos naturais devido ao uso crescente de agrotóxicos na cultura. Sendo assim, o objetivo deste trabalho foi avaliar o efeito que diferentes agrotóxicos causam na emergência de $\boldsymbol{T}$. remus quando aplicados nas fases de larva e pupa.

\section{MATERIAL E MÉTODOS}

O trabalho foi conduzido segundo metodologia de BUENO et. al. (2008a) no laboratório de Entomologia da Embrapa Arroz e Feijão, Santo Antônio de Goiás, Goiás (GO), com cartelas contendo posturas de 100 a 150 ovos de $\boldsymbol{S}$. frugiperda, parasitadas por T. remus, seis e 10 dias após o parasitismo (fase de larva e pupa, respectivamente) (BUENO et al., 2008b) e 10 repetições por tratamento. Os ovos parasitados foram imersos por cinco segundos nas caldas pesticidas, preparadas para um volume de 
aplicação de 200L ha-1, objetivando garantir uma exposição máxima do inimigo natural aos efeitos dos agrotóxicos testados. A máxima exposição permite concluir que os produtos seletivos serão seletivos em qualquer condição de campo, enquanto resultados obtidos apenas a campo são sempre variáveis em função de cada condição experimental específica.

Após secagem sob papel absorvente por duas horas, as cartelas foram identificadas e mantidas em sacos plásticos transparentes $(4 \times 15 \mathrm{~cm})$ com ar e foram acondicionadas em ambiente controlado sob temperatura de $28 \pm 1^{\circ} \mathrm{C}$, umidade relativa do ar de $70 \pm 10 \%$ e fotofase de 12 horas, até a emergência dos adultos.

A viabilidade do parasitismo (\%) foi avaliada contando-se os ovos parasitados e os que efetivamente tiveram a emergência dos parasitoides. A redução na emergência de $\boldsymbol{T}$. remus em relação ao tratamento testemunha foi calculada pela equação $\mathrm{E}(\%)=(1-\mathrm{Vt} /$ Vc)x100, em que: $E(\%)$ é a porcentagem de redução da viabilidade do parasitismo; Vt é a viabilidade do parasitismo médio para o tratamento testado; e Vc é a viabilidade do parasitismo médio observado para o tratamento testemunha. Os agrotóxicos foram classificados de acordo com as normas padronizadas pela International Organization of Biological Control (IOBC) em: classe 1 - inócuo $(\mathrm{E}<30 \%)$; classe 2 levemente nocivo $(30 \leq \mathrm{E} \leq 79 \%)$; classe 3 moderadamente nocivo ( $80 \leq \mathrm{E} \leq 99 \%)$; classe 4 - nocivo (E>99\%) (HASSAN, 1992; MANZONI et al., 2007). Os produtos testados e seus respectivos nomes comerciais estão na tabela 1 . Os resultados obtidos foram submetidos às análises exploratórias para avaliar as pressuposições de normalidade dos resíduos, a homogeneidade de variância dos tratamentos e a aditividade do modelo para permitir a aplicação da

Tabela 1 - Agrotóxicos utilizados na cultura da soja (AGROFIT, 2008) e avaliados nos testes de seletividade aos estágios imaturos de Telenomus remus, após preparados para um volume de calda de $200 \mathrm{~L} \mathrm{ha}^{-1}$.

\begin{tabular}{|c|c|c|c|c|c|}
\hline Ingrediente ativo & Nome comercial & Tipo $^{1}$ & Grupo químico & $\mathrm{DI}^{2}$ & $\mathrm{DC}^{3}$ \\
\hline Acefato & Acefato Nortox & I & Organofosforado & 525 & 0,7 \\
\hline Bifentrina & Talstar 100EC & I & Piretroide & 5 & 0,05 \\
\hline Clorpirifós & Lorsban 480BR & I & Organofosforado & 384 & 0,8 \\
\hline Diflubenzurom & Dimilin 80WG & I & Benzoiluréia & 20 & 0,08 \\
\hline Espinosade & Tracer & I & Espinosinas & 24 & 0,05 \\
\hline Flufenoxurom & Cascade 100 & I & Benzoiluréia & 10 & 0,1 \\
\hline Gama-cialotrina & Stallion150CS & I & Piretroide & 3,75 & 0,025 \\
\hline Imidacloprido + beta-ciflutrina & Connect & I & Neonicotinóide + piretróide & $100+12,5$ & 1 \\
\hline Metoxifenozido & Intrepid 240SC & I & Diacilhidrazina & 21,6 e 36 & 0,09 e 0,15 \\
\hline $2,4 \mathrm{D}$ & DMA 806BR & $\mathrm{H}$ & Ácido ariloxialcanóico & 1209 & 1,5 \\
\hline Clomazona & Gamit & $\mathrm{H}$ & Isoxazolidinona & 1000 & 2 \\
\hline Dicloreto de paraquate & Gramoxone 200 & $\mathrm{H}$ & Bipiridílio & 600 & 3 \\
\hline Diurom + dicloreto de paraquate & Gramocil & $\mathrm{H}$ & Uréia + bipiridílio & $300+600$ & 3 \\
\hline Flumioxazina & Flumyzin 500 & $\mathrm{H}$ & Ciclohexenodicarboximida & 60 & 0,12 \\
\hline Glifosato & Roundup Ready & $\mathrm{H}$ & Glicina substituída & 1200 & 2,5 \\
\hline Glifosato & Roundup Transorb & $\mathrm{H}$ & Glicina substituída & 2592 & 4 \\
\hline Glifosato & Gliz 480SL & $\mathrm{H}$ & Glicina substituída & 2880 & 6 \\
\hline Glifosato + imazetapir & Alteza 30SL & $\mathrm{H}$ & Glicina substituída + imidazolinona & $720+90$ & 3 \\
\hline S-metolacloro & Dual Gold & $\mathrm{H}$ & Cloroacetanilida & 1920 & 2 \\
\hline Azoxistrobina & Priori & $\mathrm{F}$ & Estrobilurina & 50 & 0,2 \\
\hline Azoxistrobina + ciproconazole & Priori Xtra & $\mathrm{F}$ & Estrobilurina + triazol & $60+24$ & 0,3 \\
\hline Carbendazin & Derosal 500SC & $\mathrm{F}$ & Benzimidazol & 250 & 0,5 \\
\hline Epoxiconazole & Opus SC & $\mathrm{F}$ & Triazol & 12,5 & 0,1 \\
\hline Epoxiconazole + piraclostrobina & Opera & $\mathrm{F}$ & Triazol + estrobilurina & $30+79,8$ & 0,6 \\
\hline Flutriafol & Impact & $\mathrm{F}$ & Triazol & 125 & 1 \\
\hline Flutriafol + tiofanato metílico & Celeiro & $\mathrm{F}$ & Triazol + benzimidazol & $60+300$ & 0,6 \\
\hline Tebuconazole & Folicur EC & $\mathrm{F}$ & Triazol & 150 & 0,75 \\
\hline Tiofanato metílico & Cercobin 500SC & $\mathrm{F}$ & Benzimidazol & 400 & 0,8 \\
\hline Trifloxistrobina + tebuconazole & Nativo & $\mathrm{F}$ & Estrobilurina + triazol & $60+120$ & 0,6 \\
\hline
\end{tabular}

${ }^{1} \mathrm{I}=$ inseticida, $\mathrm{F}$ = fungicida, $\mathrm{H}=$ herbicida; ${ }^{2} \mathrm{DI}=$ Dosagem do ingrediente ativo em g ha ${ }^{-1}$ ou g $200 \mathrm{~L}^{-1}$; ${ }^{3} \mathrm{DC}=\mathrm{Dosagem}$ da formulação comercial em L ou kg ha-1 ou L ou kg 200L-1. 
ANOVA. Quando necessário, os dados foram transformados em $\log \mathrm{X}$. As médias foram comparadas pelo teste de Tukey, a 5\% de probabilidade de erro.

\section{RESULTADOS E DISCUSSÃO}

A viabilidade do parasitismo observada nos tratamentos com flufenoxurom 10, diflubenzurom 20, metoxifenozido 21,60 e $36 \mathrm{~g}$ i.a. ha ${ }^{-1}$ foi igual ou superior a $80 \%$ e estatisticamente igual ao tratamento testemunha (Tabela 2). Portanto, esses produtos foram classificados como inócuos (classe 1) a ambas as fases imaturas (larva e pupa) de T. remus nas respectivas doses testadas (Tabela 3). Flufenoxurom e diflubenzurom são inseticidas reguladores de crescimento do grupo das benzoilfeniluréias que inibem a síntese de quitina (REYNOLDS, 1987). Metoxifenozido, outro inseticida regulador de crescimento, é um agonista de ecdisteroide do grupo das diacilhidrazinas e provoca uma aceleração no processo da ecdise (DHADIALLA et al., 1998). Os inseticidas reguladores de crescimento são normalmente destacados pela sua seletividade aos agentes de controle biológico (CARVALHO et al., 1994),

Tabela 2 - Viabilidade média ( \pm EPM) de larvas e pupas de Telenomus remus após a aplicação de diferentes agrotóxicos.

\begin{tabular}{|c|c|c|c|c|}
\hline & Tratamentos (g. i.a. ha ${ }^{-1}$ ) & Larvas & Pupas & \\
\hline \multirow{11}{*}{ 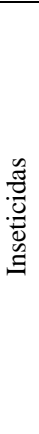 } & Acefato 525 & $84,6 \pm 6,7 \quad a^{1}$ & $77,3 \pm 7,9$ & $\mathrm{ab}$ \\
\hline & Bifentrina 5 & $43,0 \pm 11,0 \quad b$ & $54,7 \pm 8,2$ & b \\
\hline & Clorpirifós 384 & $0,0 \pm 0,0 \quad$ c & $0,0 \pm 0,0$ & c \\
\hline & Diflubenzurom 20 & $85,9 \pm 5,3 \quad a$ & $84,5 \pm 6,4$ & a \\
\hline & Espinosade 24 & $76,1 \pm 3,6 \quad$ a & $69,5 \pm 3,1$ & $\mathrm{ab}$ \\
\hline & Flufenoxurom 10 & $87,8 \pm 1,2 \quad a$ & $88,1 \pm 4,6$ & $\mathrm{a}$ \\
\hline & Gama-cialotrina 3,75 & $35,3 \pm 4,4 \quad b$ & $60,6 \pm 7,4$ & $a b$ \\
\hline & Imidacloprido 100 + beta-ciflutrina 12,5 & $86,5 \pm 3,1$ a & $84,8 \pm 6,8$ & $\mathrm{a}$ \\
\hline & Metoxifenozido 21,60 & $98,7 \pm 0,8 \quad a$ & $86,2 \pm 2,8$ & a \\
\hline & Metoxifenozido 36,00 & $80,1 \pm 2,9 \quad a$ & $79,6 \pm 5,7$ & $\mathrm{ab}$ \\
\hline & Testemunha (água) & $89,6 \pm 3,0 \quad a$ & $86,0 \pm 4,2$ & $\mathrm{a}$ \\
\hline \multirow{11}{*}{ 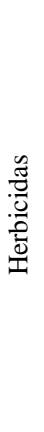 } & 2,4-D 1209 & $56,0 \pm 10,9^{\mathrm{NS}}$ & $47,2 \pm 4,5$ & $\mathrm{ab}$ \\
\hline & Clomazona 1000 & $73,3 \pm 4,8$ & $35,9 \pm 9,7$ & $\mathrm{ab}$ \\
\hline & Dicloreto de paraquate 600 & $69,2 \pm 7,6$ & $51,8 \pm 7,4$ & $a b$ \\
\hline & Dicloreto de paraquate 600 + diurom 300 & $72,3 \pm 8,3$ & $48,8 \pm 3,9$ & ab \\
\hline & Flumioxazina 60 & $66,9 \pm 8,7$ & $55,8 \pm 8,1$ & $\mathrm{a}$ \\
\hline & Glifosato 1200 (Roundup Ready®) & $67,8 \pm 8,7$ & $36,5 \pm 5,8$ & $\mathrm{ab}$ \\
\hline & Glifosato 2592 (Roundup Transorb®) & $49,7 \pm 9,1$ & $42,7 \pm 8,2$ & $\mathrm{ab}$ \\
\hline & Glifosato 2880g $\left(\mathrm{Gliz}^{\circledR}\right)$ & $68,4 \pm 8,0$ & $35,5 \pm 5,8$ & $a b$ \\
\hline & Glifosato 720 + imazetapir 90 & $79,4 \pm 7,0$ & $25,9 \pm 4,7$ & b \\
\hline & S-metolacloro 1920 & $55,0 \pm 8,8$ & $44,1 \pm 4,8$ & $\mathrm{ab}$ \\
\hline & Testemunha (água) & $68,1 \pm 4,0$ & $55,9 \pm 4,4$ & $\mathrm{a}$ \\
\hline \multirow{11}{*}{ } & Azoxistrobina 50 & $62,9 \pm 8,7^{\mathrm{NS}}$ & $47,0 \pm 5,3^{\mathrm{NS}}$ & \\
\hline & Azoxistrobina 60 + ciproconazole 24 & $74,8 \pm 5,4$ & $71,6 \pm 4,8$ & \\
\hline & Carbenzadim 250 & $70,0 \pm 13,1$ & $54,9 \pm 5,8$ & \\
\hline & Epoxiconazole 12,5 & $68,9 \pm 10,7$ & $49,9 \pm 8,8$ & \\
\hline & Epoxiconazole 30 + piraclostrobina 79,8 & $57,1 \pm 9,2$ & $53,9 \pm 5,9$ & \\
\hline & Flutriafol 125 & $57,9 \pm 12,5$ & $47,7 \pm 3,6$ & \\
\hline & Flutriafol 60 + tiofanato-metílico 300 & $65,6 \pm 3,4$ & $66,6 \pm 4,4$ & \\
\hline & Tebuconazole 150 & $51,8 \pm 7,2$ & $59,5 \pm 7,7$ & \\
\hline & Tiofanato-metílico 400 & $44,0 \pm 10,6$ & $69,3 \pm 10,8$ & \\
\hline & Trifloxistrobina 60 + tebuconazole 120 & $67,5 \pm 7,4$ & $56,3 \pm 8,1$ & \\
\hline & Testemunha (água) & $89,1 \pm 3,2$ & $76,0 \pm 8,7$ & \\
\hline
\end{tabular}

\footnotetext{
${ }^{1}$ Médias seguidas pela mesma letra, em cada fase de desenvolvimento e tipo de agrotóxico (inseticida, herbicida ou fungicida), não diferem estatisticamente entre si pelo teste de Tukey, a 5\% de probabilidade. ${ }^{\mathbf{N}}$ Diferença não significativa. Antes da análise, os dados foram transformados em Log (X) sempre quando necessário.
}

Ciência Rural, v.39, n.8, nov, 2009. 
Tabela 3 - Efeito de diferentes agrotóxicos (E) sobre larvas (ovos parasitados de seis dias) e pupas (ovos parasitados de 10 dias) de Telenomus remus, após imersão nos tratamentos por cinco segundos.

\begin{tabular}{|c|c|c|c|c|c|}
\hline & \multirow{2}{*}{ Tratamentos (g. i.a. ha ${ }^{-1}$ ) } & \multicolumn{2}{|c|}{ Larvas } & \multicolumn{2}{|c|}{ Pupas } \\
\hline & & E (\%) & Classe $^{1}$ & E (\%) & Classe $^{1}$ \\
\hline \multirow{10}{*}{ 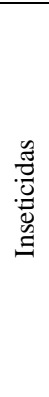 } & Acefato 525 & 5,6 & 1 & 10,1 & 1 \\
\hline & Bifentrina 5 & 52,1 & 2 & 36,4 & 2 \\
\hline & Clorpirifós 384 & 100 & 4 & 100 & 4 \\
\hline & Diflubenzurom 20 & 4,1 & 1 & 1,7 & 1 \\
\hline & Espinosade 24 & 15,0 & 1 & 29,6 & 1 \\
\hline & Flufenoxurom 10 & 2,0 & 1 & 0 & 1 \\
\hline & Gama-cialotrina 3,75 & 60,6 & 2 & 29,6 & 1 \\
\hline & Imidacloprido 100 + beta-ciflutrina 12,5 & 3,4 & 1 & 1,4 & 1 \\
\hline & Metoxifenozido 21,60 & 0 & 1 & 0 & 1 \\
\hline & Metoxifenozido 36,00 & 10,6 & 1 & 7,4 & 1 \\
\hline \multirow{10}{*}{ 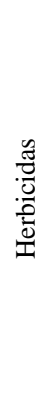 } & 2,4-D 1209 & 17,8 & 1 & 15,5 & 1 \\
\hline & Clomazona 1000 & 0 & 1 & 35,9 & 2 \\
\hline & Dicloreto de paraquate 600 & 0 & 1 & 7,3 & 1 \\
\hline & Dicloreto de paraquate $600+$ diurom 300 & 0 & 1 & 12,8 & 1 \\
\hline & Flumioxazina 60 & 1,8 & 1 & 0,2 & 1 \\
\hline & Glifosato 1200 (Roundup Ready ${ }^{\circledR}$ ) & 0,5 & 1 & 34,8 & 2 \\
\hline & Glifosato 2592 (Roundup Transorb ${ }^{\circledR}$ ) & 27,0 & 1 & 23,7 & 1 \\
\hline & Glifosato 2880g $\left(\mathrm{Gliz}^{\circledR}\right)$ & 0 & 1 & 36,6 & 2 \\
\hline & Glifosato $720+$ imazetapir 90 & 0 & 1 & 53,7 & 2 \\
\hline & S-metolacloro 1920 & 19,3 & 1 & 21,1 & 1 \\
\hline \multirow{10}{*}{ 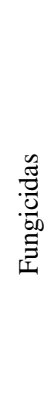 } & Azoxistrobina 50 & 29,5 & 1 & 38,2 & 2 \\
\hline & Azoxistrobina 60 + ciproconazole 24 & 16,1 & 1 & 5,8 & 1 \\
\hline & Carbenzadim 250 & 21,4 & 1 & 27,7 & 1 \\
\hline & Epoxiconazole 12,5 & 22,7 & 1 & 34,3 & 2 \\
\hline & Epoxiconazole 30 + piraclostrobina 79,8 & 35,9 & 2 & 29,1 & 1 \\
\hline & Flutriafol 125 & 35,0 & 2 & 37,2 & 2 \\
\hline & Flutriafol 60 + tiofanato-metílico 300 & 26,4 & 1 & 12,4 & 1 \\
\hline & Tebuconazole 150 & 41,9 & 2 & 21,8 & 1 \\
\hline & Tiofanato-metílico 400 & 50,6 & 2 & 8,8 & 1 \\
\hline & Trifloxistrobina $60+$ tebuconazole 120 & 24,3 & 1 & 25,9 & 1 \\
\hline
\end{tabular}

${ }^{1}$ Classe 1 - inócuo (E<30\%), classe 2 - levemente nocivo (30 $\leq \mathrm{E} \leq 79 \%$ ), classe 3 - moderadamente nocivo (80 $\leq \mathrm{E} \leq 99 \%$ ), classe 4 - nocivo $(\mathrm{E}>99 \%)$.

o que foi ratificado com os resultados obtidos neste trabalho, já que a seletividade a larvas e pupas de $\boldsymbol{T}$. remus foi uma característica comum a todos os inseticidas testados desse grupo. Entretanto, estudos semelhantes realizados com Trichogramma pretiosum, utilizando como hospedeiro alternativo Anagasta kuehniella, mostraram que inseticidas reguladores de crescimento podem, algumas vezes, ser classificados como nocivos, dependendo do produto e da dose testados e da fase de desenvolvimento do parasitoide utilizada no bioensaio (BUENO et al., 2008a). De acordo com PRATISSOLI et al. (2004), lufenurom, outro inseticida do grupo dos regulares de crescimento, ocasionou redução no parasitismo por T. pretiosum quando comparado ao tratamento testemunha. Esse contraste pode estar relacionado com as diferenças existentes entre as espécies de parasitoides testados e entre os ovos dos hospedeiros, e os ovos de $\boldsymbol{S}$. frugiperda, além de serem mais espessos que os de Anagasta kuehniella, por exemplo, podem estar dispostos em posturas realizadas em camadas sobrepostas, o que pode estar dificultando a penetração e ação dos agrotóxicos. Essa possibilidade precisa ainda ser avaliada em novas pesquisas sobre 0 assunto.

Os inseticidas acefato 525 e imidacloprido 100 + beta-ciflutrina 12,5 g i.a. ha ${ }^{-1}$ também não reduziram 
a viabilidade do parasitismo de $\mathbf{T}$. remus em relação à testemunha (Tabela 2), sendo classificados como inócuos (classe 1) para larvas e pupas do parasitoide (Tabela 3). Ambos são produtos que agem por contato e ingestão, atingindo e desorganizando o sistema nervoso dos insetos. Resultados diferentes foram obtidos por ROCHA \& CARVALHO (2004), os quais verificaram que o acefato foi prejudicial ao parasitismo de T. pretiosum, causando redução superior a $80 \%$, sendo classificado como classe 3 . Essas diferenças ressaltam a importância de estudar a seletividade dos diferentes produtos fitossanitários às mais diversas espécies de agentes de controle biológico, uma vez que a ação desses agrotóxicos pode ser diferente para cada espécie, como já discutido neste trabalho.

Larvas e pupas de T. remus, quando tratadas com bifentrina 5g. i.a. ha ${ }^{-1}$, tiveram uma viabilidade do parasitismo (\%) estatisticamente inferior ao tratamento testemunha (Tabela 2), com uma redução de 52,1 e 36,4\% para larvas e pupas, respectivamente. Esse tratamento foi classificado como levemente nocivo (classe 2) (Tabela 3). Gama-cialotrina 3,75g. i.a. ha ${ }^{-1}$ também foi classificado como levemente nocivo (classe 2) para larvas de T. remus. Porém, a fase de pupa foi ligeiramente mais tolerante, sendo o produto classificado como inócuo (classe 1) para esta fase (Tabela 3). Bifentrina e gama-cialotrina são inseticidas do grupo dos piretroides que são químicos neurotóxicos que agem no sistema nervoso do inseto, paralisando e matando rapidamente esses insetos (BUENO et al., 2008a). Inseticidas desse grupo são geralmente classificados como produtos pouco seletivos aos inimigos naturais (CARVALHO et al., 1999). BUENO et al. (2008a) e MANZONI et al. (2007) mostraram o efeito nocivo de alguns inseticidas piretroides ao parasitoide de ovos $T$. pretiosum e Trichogramma atopovilia, respectivamente.

Espinosade 24g i.a. ha-1 foi seletivo à $\boldsymbol{T}$. remus na fase de larva (classe 1) (Tabela 3), e a viabilidade do parasitismo foi igual à testemunha (Tabela 2). O espinosade é um inseticida de origem natural, oriundo da fermentação da bactéria Sacharopolyspora spinosa, sendo um produto fitossanitário aceito na agricultura orgânica nos Estados Unidos (WILLIAMS et al., 2003). Resultados diferentes foram obtidos por BUENO et al. (2008a), estudando a seletividade do espinosade ao $\boldsymbol{T}$. pretiosum. Entretanto, é importante ressaltar que essas diferenças podem se dever ao ovo do hospedeiro e à espécie do parasitoide que foram diferentes nesses estudos. Clorpirifós foi nocivo às fases de larva e pupa de T. remus, causando uma redução de $100 \%$ na viabilidade do parasitismo (\%) (Tabela 2), o que fez com que esse produto fosse classificado como nocivo (classe 4) (Tabela 3). HOHMANN, (1991 e 1993) e BUENO et al. (2008a) relataram resultados menos danosos do clorpirifós ao parasitoide T. pretiosum, porém quando testado em doses inferiores à dose utilizada neste estudo.

Com relação aos herbicidas testados, 2,4-D

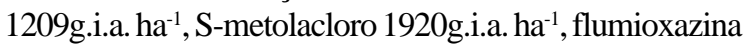
60 g.i.a. ha ${ }^{-1}$, dicloreto de paraquate $600+$ diurom 300 g.i.a. ha $^{-1}$, dicloreto de paraquate 600 g.i.a. ha ${ }^{-1}$ e glifosato 2592g.i.a. ha- ${ }^{-1}$ Roundup Transorb $^{\circledR}$ ) não reduziram a viabilidade do parasitismo em relação ao tratamento testemunha para ambas as fases testadas do parasitoide (Tabela 2), sendo classificados como seletivos (classe 1) (Tabela 3). Entretanto, glifosato $720+$ imazetapir 90 g.i.a. ha ${ }^{-1}$, clomazona 1000 g.i.a. ha ${ }^{-1}$, glifosato 2880g.i.a. ha ${ }^{-1}\left(\mathrm{Gliz}^{\circledR}\right.$ ) e glifosato 1200 g.i.a. ha ${ }^{-1}$ (Roundup Ready $^{\circledR}$ ) foram seletivos para a fase de larva e levemente nocivos (classe 2) para a fase de pupa de $\mathbf{T}$. remus (Tabela 3). Entre esses herbicidas, apenas a viabilidade de pupas de T. remus, após tratadas com glifosato 720 + imazetapir 90g.i.a. ha ${ }^{-1}$, foi inferior ao tratamento testemunha (Tabela 2). Resultados semelhantes foram relatados por STEFANELLO JÚNIOR et al (2008) com o glifosato 2880g. i.a. ha ${ }^{-1}$ para T. pretiosum. Entretanto, os mesmos autores, estudando uma outra formulação de glifosato (Roundup Transorb), obtiveram a classificação 3 (moderadamente nocivo), o que diferiu da classe 1 (inócuo) encontrada neste trabalho para o mesmo Roundup Transorb (Tabela 3). Segundo GIOLO et al. (2005), o tipo de sal presente na formulação do glifosato se constitui em fator preponderante para a toxicidade à forma adulta de Trichogramma spp. Essa diferença não foi obsevada para $T$. remus, o que ressalta mais uma vez a importância do estudo de seletividade para diferentes agentes de controle biológico. Os resultados obtidos neste estudo fornecem indícios que a espécie $T$. remus é menos sensível à ação dos herbicidas que o $\mathbf{T}$. pretiosum.

Os fungicidas avaliados também apresentaram resultados classificados entre inócuo (classe 1) e levemente nocivo (classe 2), dependendo do produto e da dose avaliados, assim como a fase de desenvolvimento do inseto. Flutriafol $60+$ tiofanatometílico 300g.i.a. ha ${ }^{-1}$, carbenzadim 250g.i.a. ha ${ }^{-1}$, tebuconazole 120 + trifloxistrobina 60g.i.a. ha ${ }^{-1} \mathrm{e}$ azoxistrobina 50 + ciproconazole 24g.i.a. ha ${ }^{-1}$ foram classificados como inócuos (classe 1) (Tabela 3). Tiofanato-metílico 400g.i.a. ha ${ }^{-1}$, tebuconazole 150 g.i.a. ha $^{-1}$ e epoxiconazole 30 + piraclostrobina 79,8g.i.a. ha ${ }^{-1}$ foram levemente nocivos (classe2) para larvas e inócuos (classe 1) para pupas de T. remus (Tabela 3). De maneira inversa, epoxiconazole 12,5g.i.a. ha ${ }^{-1}$ e 
azoxistrobina 50g.i.a. ha ${ }^{-1}$ foram seletivos (classe 1 ) para a fase de larva e levemente nocivos (classe 2) para a fase de pupa do parasitoide (Tabela 3). Flutriafol foi classificado como levemente nocivo (classe 2) para ambas as fases de T. remus (Tabela 3). Os resultados apresentados mostram que os fungicidas pouco afetam as populações de $\boldsymbol{T}$. remus. Isso é importante principalmente ao considerar que aplicações de fungicidas, as quais quase não eram realizadas antes da ocorrência da ferrugem-asiática da soja, passaram a ser comum após sua ocorrência e podem estar afetando direta ou indiretamente os inimigos naturais. BUENO et al., (2008a) relatou que o fungicida tebuconazole 200 + trifloxistrobina $100 \mathrm{~g}$ i.a. ha ${ }^{-1}$ foi nocivo (classe 4) à fase de ovo de $\boldsymbol{T}$. pretiosum, o que não foi observado para T. remus, que assim como para os herbicidas, também se mostrou mais tolerante à ação dos fungicidas em comparação ao T. pretiosum.

É preciso levar em consideração que os bioensaios conduzidos neste estudo foram realizados com fases protegidas, dentro dos ovos dos hospedeiros, por meio da imersão desses ovos parasitados na calda química. Isso garante a maior exposição dos parasitoides aos efeitos dos agrotóxicos testados, garantindo que os produtos considerados seletivos nesta pesquisa sejam sempre seletivos em qualquer condição de campo, não precisando ser mais testado para essa fase de desenvolvimento do inimigo natural. Por outro lado, os produtos classificados como classe 2, 3 e 4 em condições de laboratório podem também ser testados em condições de campo, para avaliar sua toxicidade ao inimigo natural nessas condições (HASSAN, 1992). Entretando, os resultados de campo serão sempre variáveis, dependendo das condições específicas de cada localidade. Fatores como a degradação dos compostos pela ação da luz podem ser diferenciados e possivelmente acelerados (ROCHA \& CARVALHO, 2004).

É importante considerar também que a fase adulta desses parasitoides é de vida livre e, normalmente, mais sensível à ação de agrotóxicos em comparação com as fases protegidas, dentro dos ovos do hospedeiro, como as testadas neste experimento (HASSAN, 1992). Sendo assim, pesquisas futuras estudando o efeito desses agrotóxicos sobre adultos de $\boldsymbol{T}$. remus ainda precisam ser realizadas, e os resultados considerados na tomada de decisão, na escolha do melhor produto para ser utilizado no MIP.

\section{CONCLUSÕES}

Os tratamentos de inseticidas acefato 525; diflubenzurom 20; flufenoxuron 10; imidacloprido 100
+ beta-ciflutrina 12,5; metoxifenozido 21,6; metoxifenozido 36 gramas de ingrediente ativo ha ${ }^{-1}$, de herbicidas 2,4-D 1209; dicloreto de paraquate 600; dicloreto de paraquate 600 + diurom 300; flumioxazina 60; glifosato 2592 (Roundup Transorb ${ }^{\circledR}$ ); S-metolacloro 1920 gramas de ingrediente ativo ha $^{-1}$ e de fungicidas azoxistrobina 60 + ciproconazole 24; carbenzadim 250; flutriafol 60 + tiofanato-metílico 300 e trifloxistrobina $60+$ tebuconazole 120 gramas de ingrediente ativo ha- ${ }^{-1}$ são seletivos às fases de larva e pupa de T.remus e podem ser utilizados dentro do MIP quando necessários, sem prejuízos para esse inimigo natural. Os demais produtos avaliados têm efeitos negativos indesejáveis em, pelo menos, um dos estágios de desenvolvimento avaliado. Assim, sua seletividade deve ser avaliada em condições de campo e sua utilização permitida apenas nas situações específicas em que a seletividade eventualmente for comprovada.

\section{AGRADECIMENTOS}

À unidade da Embrapa Arroz e Feijão, pelo apoio na realização da pesquisa. À Coordenação de Aperfeiçoamento de Pessoal de Nível Superior (CAPES) e ao Conselho Nacional de Desenvolvimento Científico e Tecnológico (CNPq), pelo apoio financeiro e pela concessão das bolsas que tornaram viáveis a realização deste trabalho. Este artigo foi revisado e aprovado para publicação pelo Comitê de Publicações da Embrapa Soja sob o número 21/2008.

\section{REFERÊNCIAS}

BUENO, A.F.; FREITAS, S. Effect of the insecticides abamectin and lufenuron on eggs and larvae of Chrysoperla externa under laboratory conditions. Biocontrol, v.39,p.277-283, 2004. Disponível em: $<$ http://www.springerlink.com/content/g5486t377874u324/>. Acesso em: 24 jun. 2009. doi: 10.1023/B:BICO.0000025375.07428.0b.

BUENO, A.F. et al. Effects of pesticides used in soybean crops to the egg parasitoid Trichogramma pretiosum. Ciência Rural, v.38, n.6, p.1495-1503, 2008a. Disponível em: <http:/ /www.scielo.br/scielo.php?script=sci_arttext\&pid=S010384782008000600001\&lng=en\&nrm=iso>. Acesso em: 24 jun. 2009. doi: 10.1590/S0103-84782008000600001.

BUENO, R.C.O.F. et al. Biology and thermal requirements of Telenomus remus reared on fall armyworm Spodoptera frugiperda eggs. Ciência Rural, v.38, n.1, p.1-6, 2008b. Disponível em: <http://www.scielo.br/scielo.php?script=sci_arttext\&pid=S010384782008000100001\&lng=en\&nrm=iso>. Acesso em: 24 jun. 2009. doi: 10.1590/S0103-84782008000100001.

CARVALHO, G.A. et al. Seletividade de inseticidas reguladores de crescimento de insetos à Trichogramma pretiosum Riley (Hymenoptera: Trichogrammatidae). Anais da Sociedade Entomológica do Brasil, v.23, n.3, p.431-434, 1994.

CARVALHO, G.A. et al. Ação residual de alguns inseticidas pulverizados em plantas de tomateiro sobre duas linhagens de Trichogramma pretiosum Riley, 1879 (Hymenoptera: 
Trichogrammatidae) em casa-de-vegetação. Ciência e Agrotecnologia, v.23, n.4, p.770-775, 1999.

CAVE, R.D. Biology, ecology and use in pest management of Telenomus remus. Biocontrol News and Information, v.21, n.1, p.21-26, 2000.

CONAB (Brasília, DF). Safra 2007/2008: sétimo levantamento Abril/2008. Disponível em: <www.conab.gov.br> Acesso em: 25 de abril. 2008.

DHADIALLA, T.S. et al. New insecticides with ecdysteroidal and juvenile hormone activity. Annual Review of Entomology, v.43, p.545-569, 1998. Disponível em: <http:/ / a r journals.annualreviews.org/doi / abs / 10.1146\%2Fannurev.ento.43.1.545>. Acesso em: 24 jun. 2009. doi:10.1146/annurev.ento.43.1.545.

DIEZ-RODRÍGUEZ, G.I.; OMOTO, C. Herança da resistência de Spodoptera frugiperda (J.E. Smith) (Lepidoptera: Noctuidae) a lambda-cialotrina. Neotropical Entomology, v.30, n.2, p.311-316, 2001.

FERRER, F. Biological of agricultural insect pest in Venezuela; advances, achievements, and future perspectives. Biocontrol News and Information, v.22, n.3, p. 67-74, 2001.

GIOLO, F.P. et al. Seletividade de formulações de glyphosate a Trichogramma pretiosum (Hymenoptera: Trichogrammatidae). Planta Daninha, v.23, p.457-462, 2005. Disponível em: $<$ http://www.scielo.br/scielo.php?pid=S 0100 83582008000200010\&script=sci_arttext $>$. Acesso em: 24 jun. 2009. doi: 10.1590/S0100-83582008000200010.

HASSAN, S.A. Guidelines for testing the effects of pesticides on beneficials organisms: description of test methods. In: Guidelines for testing the effects of pesticides on beneficials organism. International Union of Biological Sciences. 1992. p.18- 39. (Bulletin OILB/SROP 1992/XV/3).

HOHMANN, C.L. Efeito de alguns inseticidas sobre adultos de Trichogramma pretiosum RILEY. Anais da Sociedade Entomológica do Brasil, v.22, n.3, p.563-567, 1993.

HOHMANN, C.L. Efeito de diferentes inseticidas sobre a emergência de Trichogramma pretiosum (Hymenoptera: Trichogrammatidae). Anais da Sociedade Entomológica do Brasil, v.20, n.1, p.59-65, 1991.

MANZONI, C.G. et al. Seletividade de Agroquímicos utilizados na produção integrada de maçã aos parasitóides Trichogramma pretiosum Riley e Trichogramma atopovirilia Oatan \& Platner (Hymenoptera: Trichogrammatidae). Bioassay, v.2, n.1, p.111, 2007.

MORALES, J.et al. Patrón de emergência, longevidad, parasitismo y proporción sexual de Telenomus remus (Hymenoptera: Scelionidae) com relación al cogollero Del maíz. Bioagro, v.12, n.2, p. 47-54, 2000.

PALUMBO, J.C. et al. Insecticidal control and resistance management for Bemisia tabaci. Crop Protection, v.20, p.739765, 2001. Disponível em: <http://www.sciencedirect.com/ science?_ob=ArticleURL\&_udi=B6T5T-44HS98W$4 \&$ \&user $=4353287 \&$ \&doc $=1 \&$ \& fmt $=\&$ \&orig $=$ search \&_sort $=$ d\&_docanchor $=\& v i e w=c \& \_a c c t=C 0000128$ $78 \&$ _version $=1 \&$ _urlversion $=0 \&$ _userid $=4353$ 287\&md5=2f5aafc76395f0efedd295ef1aa67843>. Acesso em: 24 jun. 2009. doi:10.1016/S0261-2194(01)00117-X.

PRATISSOLI, D. et al. Ação transovariana de lufenuron (50 G/ L) sobre adultos de Spodoptera frugiperda (J. E. Smith) (Lepidoptera: Noctuidae) e seu efeito sobre o parasitóide de ovos Trichogramma pretiosum Riley (Hymenoptera: Trichogrammatidae). Ciência e Agrotecnologia, v.28, n.1, p.9-14, 2004.

REYNOLDS, S.E. The cuticule, growth regulators and moulting in insects: the essential background to the action of acylurea insecticides. Pesticide Science, v.20, p.131-146, 1987.

ROCHA, L.C.D.; CARVALHO, G.A. Adaptação da metodologia padrão da IOBC para estudos de seletividade com Trichogramma pretiosum Riley, 1879 (Hymenoptera: Trichogrammatidae) em condições de laboratório. Acta Scientiarum Agronomy, v.26, n.3, p.315-320, 2004.

VAN LENTEREN, J.C.; BUENO, V.H.P. Augmentative biological control of arthropods in Latin America. Biocontrol, v.48, p.123139, 2003. Disponível em: <http://www.springerlink.com/ content/q2722717200345h5/>. Acesso em: 24 jun. 2009. doi:10.1023/A:1022645210394.

STEFANELLO JÚNIOR, G.J. et al. Seletividade de herbicidas registrados para a cultura do milho a adultos de Trichogramma pretiosum (Hymenoptera: Trichogrammatidae). Planta Daninha, v.26, p.343-351, 2008.

WILLIAMS, T. et al. Is the naturally derived insecticide spinosad $^{\circledR}$ compatible with insect natural enemies? Biocontrol Science and Technology. v.13, n.5, 459-475, 2003. 